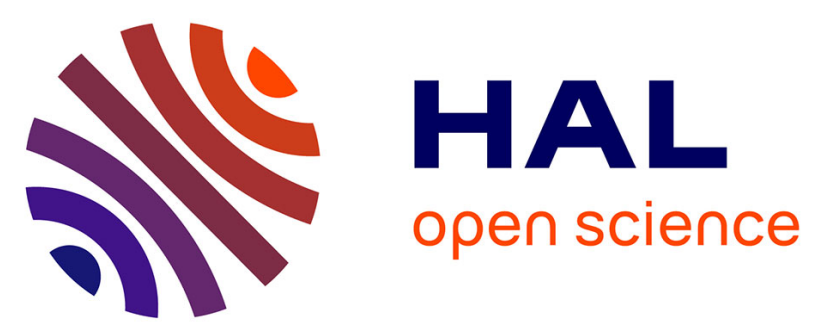

\title{
Computational Contact Problems: Investigations on the Robustness of Generalized Newton Method, Fixed-Point method and Partial Newton Method
}

\author{
A.D. Kudawoo, Micheline Abbas, T. De-Soza, Frédéric Lebon, I. Rosu
}

\section{To cite this version:}

A.D. Kudawoo, Micheline Abbas, T. De-Soza, Frédéric Lebon, I. Rosu. Computational Contact Problems: Investigations on the Robustness of Generalized Newton Method, Fixed-Point method and Partial Newton Method. International Journal for Computational Methods in Engineering Science and Mechanics, 2018, 19 (4), pp.268-282. 10.1080/15502287.2018.1502217 . hal-02021042

\author{
HAL Id: hal-02021042 \\ https://hal.science/hal-02021042
}

Submitted on 14 Jun 2019

HAL is a multi-disciplinary open access archive for the deposit and dissemination of scientific research documents, whether they are published or not. The documents may come from teaching and research institutions in France or abroad, or from public or private research centers.
L'archive ouverte pluridisciplinaire HAL, est destinée au dépôt et à la diffusion de documents scientifiques de niveau recherche, publiés ou non, émanant des établissements d'enseignement et de recherche français ou étrangers, des laboratoires publics ou privés. 


\title{
Computational Contact Problems: Investigations on the Robustness of Generalized Newton Method, Fixed-Point method and Partial Newton Method
}

\author{
A.D. Kudawoo ${ }^{a, b, c}$, M. Abbas ${ }^{b, c}$, T. De-Soza ${ }^{c}$, F. Lebon', and I. Rosu ${ }^{a}$ \\ aLaboratoire de Mécanique et d'Acoustique (LMA), CNRS UMR 7031 AMU, University of Aix-Marseille, Marseille Cedex 13, France; \\ ${ }^{\mathrm{b}}$ Institut des Sciences de la Mécanique et des applications Industrielles (IMSIA), UMR EDF-CNRS-CEA, Palaiseau, France; ${ }^{\circ}$ Electricité de \\ France Recherche et Développement-EDF Lab Saclay, Département ERMES, Palaiseau, France
}

\begin{abstract}
Dealing with frictional contact simulations, one can use generalized Newton method or Fixed-point method. In this article, we present partial Newton method which combines advantages from both algorithms. The solution techniques presented are based on an equivalent writing of the augmented Lagrangian formulation. Level-set functions are introduced to describe the status of the point. We consider various numerical examples espe-cially a large scale industrial in order to compare the algorithms implemented.
\end{abstract}

KEYWORDS Optimzation methods; contact and friction; augmented Lagrangian; robustness; performance

\section{Introduction}

\subsection{Variational formulations}

In the field of computational contact mechanics, there exist many types of variational principles and one must choose the most appropriate to come over the problem. This choice is not trivial because each formulation has its advantages and its drawbacks. It can be considered variational inequalities or variational equaslities formulations.

Variational inequalities can be found in primal forms [1-4]) or in mixed forms [2]. Recently, dual variational inequalities were developed based on Airy's functions by Kuss-Lebon [5]. The solution techniques based on these formulations are, for example, a block-relaxation procedure with projection which is detailed in [6] or a fixed-point method (FPM) described in [4] or active set strategies in $[7,8]$. We will use in this article, a GCP algorithm developed by Renouf and Alart [9] for various comparisons.

In variational equalities, it is sufficient to write rigorously the frictional-contact energy with nonsmooth operators in order to check during the resolution of Signorini-Coulomb's condition and the equilibrium state simultaneously.

A well-known mixed formulation is the so-called augmented Lagrangian formulation which is C-1 differentiable [10]. In this article, we use an equivalent form of the augmented Lagrangian formulation. It is introduced the nonsmooth operators called "level-sets" in order to treat separately the augmented pressures and the frictional-contact status. This key idea was primarily introduced by Bendhia-Zarroug [11]. Nodeto-segments or point-to-segment contact elements are used to discretize the contact area and a collocation method based on the Dirac-type functions is used for the discretization of the level-set functions. The formulation proposed reduce the Coulomb's disc with unknown radius to a reduced Coulomb's disc with radius one. One advantage of this formulation is the fact that six regularization parameters can be used during the computation, while two regularization parameters are allowed in the Alart-Curnier formulation. Multiple regularization parameters allow more control on the robustness of the GNM. Indeed, two parameters can control the frictional contact status, two parameters can control the condition number of the augmented matrices and finally two parameters can be used to control the Signorini-Coulomb's law [12].

\subsection{Numerical procedures for augmented Lagrangian formulation}

Optimization methods like the generalized Newton method (GNM) can be applied by using the 
mathematical definition of generalized Jacobian for nonsmooth operators. In other hand, one can use FPM to approximate the Coulomb's friction law by a sequence of Tresca's friction law (see Figure 1). We consider in the present article an extension of the FPM to geometry and contact status parameters.

To obtain accurate results, some mathematical properties must be checked on the discrete problems. The first one is the inf-sup conditions to avoid oscillations on the approximated solution of Lagrange multiplier. To satisfy these properties, we must focus on the space approximation for the unknowns and the numerical integration for the frictional-contact terms $[13,14]$. In this article, we consider point-to-segment contact elements which gives good results for the numerical tests exhibited. The proof of the existence and the uniqueness of the solution still remains a domain of interest. There exists a unique solution for Signorini's problem. In frictional case, the proof of the uniqueness is not trivial. It depends on many physical parameters like friction coefficients or Poisson ratios [15-17]. If the nonsmooth operators used to satisfy the homeomorphism (injectivity and surjectivity), then the solution is unique. This work was sketched by Alart [18]. Indeed, we can proove the uniqueness of the solution if some assumptions on the generalized Jacobian are satisfied [18] (the nonsmooth operators are Lipschitz continuous and cone-wise linear).

To end discussion on the mathematical properties, one must be careful on the choice of the regularization parameters. Their influences on the convergence of the GNM are proved in $[19,20]$. To improve the robustness of the numerical treatment, an original local treatment is proposed in [21] and will be briefly sketched at the end of this article.

\subsection{Summary of the article}

The next section is dedicated to the variational formulation and the discretization aspects. Then, we exhibit the solution techniques developed such as FPM, PNM, and GNM. The first numerical test shows the influence of the regularization parameter on GNM and FPM. The so-called ring-on-block test and 3Dironing test are exhibited in Sections 5 and 6 to stress the influence of friction and material nonlinearities. In the 3D-ironing test, we compare the algorithm developed with a GCP one presented in [9]. A large scale of $120,000 \mathrm{dof}^{\prime} \mathrm{s}$ is considered to stress the advantages of the PNM. Finally, Section 7 is a discussion on the solution techniques developed taking into account our various experiences in two- and three-dimensional "large-scale problems."

\section{Variational formulation and discretization aspects}

Let us recall in this section, the variational framework in the context of the quasi-static evolution, large deformations, large slips, and nonlinear materials.

It is used an inward unit normal associated with the master body $n^{m}\left(x^{m}\left(X^{m}, t\right)\right)$. The master surface is described by an Eulerian kinematical technique and the slave surface is described by a Lagrangian kinematical technique. We denote the normal gap by $d_{n}$ and the tangential gap by $d_{t}$.

Signorini-Coulomb's law is formulated as follows:

$$
\left\{\begin{array}{l}
(\text { Signorini }) \\
d_{n}=\left(\widetilde{\mathbf{x}}^{2}-\mathbf{x}^{1}\right) \cdot \mathbf{n}\left(\widetilde{\mathbf{x}}^{2}\right) \leq 0 \\
p_{n} \leq 0 \\
p_{n} d_{n}=0
\end{array}\right.
$$
complementarity
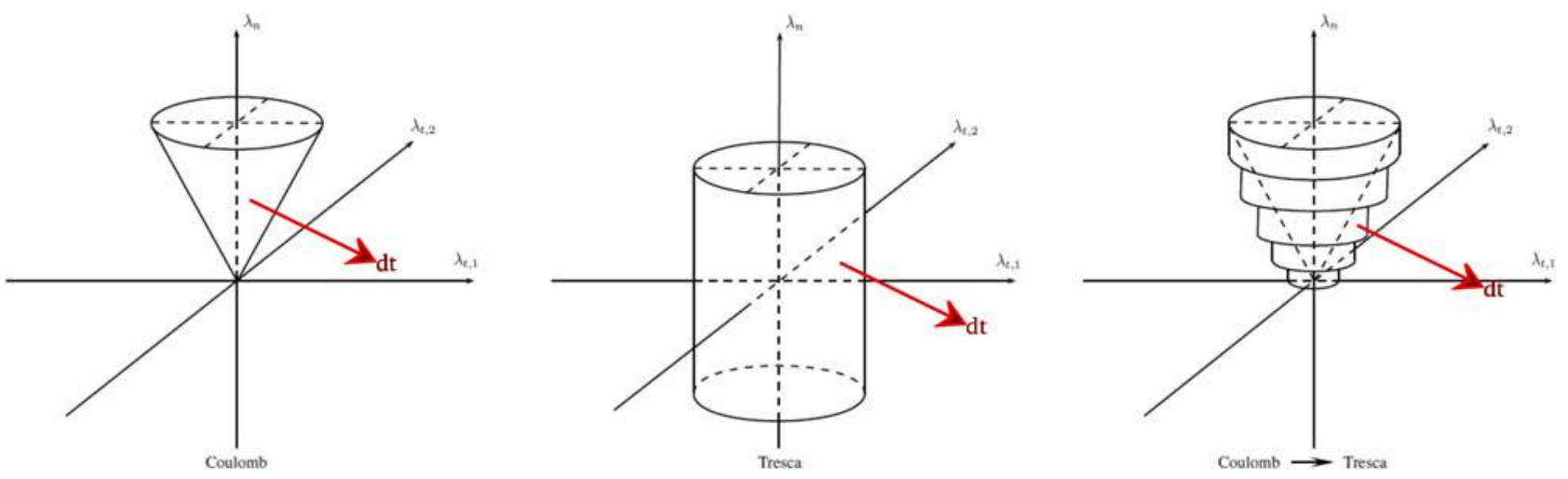

Figure 1. Coulomb and Tresca friction threshold. 


$$
\left\{\begin{array}{l}
(\text { Coulomb }) \\
\dot{\mathbf{d}}_{\mathbf{t}}=0 \quad \text { if } \| \mathbf{p}_{\mathbf{t}}||<\mu\left|p_{n}\right| \quad \text { adherence } \\
\dot{\mathbf{d}}_{\mathbf{t}}=-\alpha \mathbf{p}_{\mathbf{t}} \text { if }\left\|\mathbf{p}_{\mathbf{t}}\right\|=\mu\left|p_{n}\right| \text { with } \quad \alpha>0 \quad \text { slip } \\
\dot{\mathbf{d}}_{\mathbf{t}}\left(\left\|\mathbf{p}_{\mathbf{t}}\right\|-\mu\left|p_{n}\right|\right)=0 \quad \text { complementarity. }
\end{array}\right.
$$

\subsection{Augmented Lagrangian formulations and discretization}

The saddle-point problem consists in finding $\left(u^{1}, u^{2}, \lambda_{n}, \lambda_{t}\right)^{T}$ such that

$$
\left(u^{1}, u^{2}, \lambda_{n}, \lambda_{t}\right)=\arg \min _{\left(v^{1}, v^{2}\right)} \arg \max _{\left(\lambda_{n}^{*}, \lambda_{t}^{*}\right)}\left(\phi^{\text {total }}\left(v^{1}, v^{2}, \lambda_{n}^{*}, \lambda_{t}^{*}\right)\right),
$$

where $\phi^{\text {total }}$ is incremental contact energy associated with the mechanical system.

$$
\phi^{\text {total }}=\phi^{\text {int }}+\phi^{\text {cont }}+\phi_{C\left(\lambda_{n}\right)}^{\text {fric }}-\phi^{\text {ext }},
$$

where

$$
\begin{aligned}
& \phi^{\text {int }}=\sum \int_{\Omega_{i}} \Psi_{i}^{\text {int }}{ }_{i}(u) \mathrm{d} \Omega, \\
& \phi^{\text {cont }}=\int_{\Gamma_{c}} \Psi^{\text {cont }}\left(d_{n}, \lambda_{n}\right) \mathrm{d} \Gamma, \\
& \phi_{C\left(\lambda_{n}\right)}^{\text {fric }}=\int_{\Gamma_{c}} \Psi_{C\left(\lambda_{n}\right)}^{\text {fric }}\left(d_{t}, \lambda_{t}\right) \mathrm{d} \Gamma, \\
& \phi^{\text {ext }}=\int_{\Gamma_{f}} \Psi^{\text {ext }} \mathrm{d} \Gamma .
\end{aligned}
$$

where $\Psi^{i n t}(u)$ is the density of the energy deformation. This expression is similar to those given in Section 2, $\Psi^{\text {ext }}$ is the density of energy associated with a prescribed surface traction, $\Psi^{\text {cont }}\left(d_{n}\right), \Psi_{C\left(\lambda_{n}\right)}^{\text {fric }}\left(d_{t}\right)$ are the densities of the frictional-contact energy, and $\Psi^{\text {cont }}\left(d_{n}\right), \Psi_{C\left(\lambda_{n}\right)}^{\text {fric }}\left(d_{t}\right)$ can be formulated by the mean of indicator functions. It can also be used regularized forms of penality method. Penality method is not an exact formulation and it is not robust due to the dependence of the solution on the regularization parameter. Then, we use the well-known penality-duality method of augmented Lagrangian method.

In this article, an equivalent form the augmented formulation introduced in $[10,22]$ is used. We give its explicit form:

$\Phi^{\text {aug }}\left(d_{n}, d_{t}, \lambda_{\mathbf{n}}, \Lambda\right)=\int_{\Gamma_{c}} \Psi^{\text {cont }}\left(d_{n}, \lambda_{n}\right)+\int_{\Gamma_{c}}-\mu \lambda_{n} \Psi^{\text {fric }}\left(d_{t}, \lambda_{t}\right)$

with

$$
\Psi^{\text {cont }}\left(d_{n}, \lambda_{n}\right)=\frac{1}{2 r_{n}}\left\{S_{c}\left(\lambda_{n}-r_{n} d_{n}\right)\left\{\lambda_{n}-r_{n} d_{n}\right\}^{2}-\lambda_{n}^{2}\right\},
$$

$$
\begin{aligned}
& \Psi^{\text {fric }}\left(d_{t}, \lambda_{t}\right)=\frac{1}{2 r_{t}}\left\{\left(1-S_{c}\right) \Lambda . \Lambda+S_{c}\left\{S _ { f } \left\{\left[\Lambda+r_{t} v_{t} d t\right]\left[\Lambda+r_{t} v_{t} d t\right]\right.\right. \text {, }\right. \\
& -\Lambda . \Lambda\}+\left(1-S_{f}\right)\left\{\left[\frac{\Lambda+r_{t} v_{t} d t}{\left\|\Lambda+r_{t} v_{t} d t\right\|}\right]\left[\frac{\Lambda+r_{t} v_{t} d t}{\left\|\Lambda+r_{t} v_{t} d t\right\|}\right]\right. \\
& \left.\left.\left.-2|| \Lambda+r_{t} v_{t} d t||+\Lambda . \Lambda\right\}\right\}\right\},
\end{aligned}
$$

In the equations above:

1. $\lambda_{n}$ is the Lagrange multiplier related to Signorini conditions. It is homogeneous to a contact pressure.

2. $S_{c}($.$) indicates the contact status of a point$

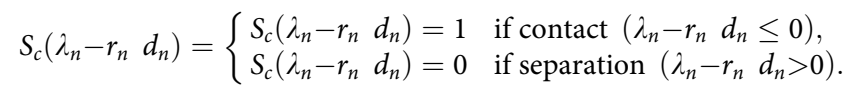

3. $\Lambda=\frac{\lambda_{t}}{-\mu \lambda_{n}}$

where $\Lambda$ is calculated from the classical Lagrange multiplier associated with Coulomb conditions. It means that the direction of the tangential contact stress is not known a priori. Using this definition, the Coulomb's disc with unknown radius $-\mu \lambda_{n}$ is reduced to a disc with known radius 1 .

4. $S_{f}($.$) indicates the slip status of a point$

$S_{f}\left(\Lambda+r_{t} d_{t}\right)=\left\{\begin{array}{ll}S_{f}\left(\Lambda+r_{t} d_{t}\right)=1 \quad \text { if adherence }\left(\left\|\Lambda+r_{t} d_{t}\right\| \leq 1\right) \\ S_{f}\left(\Lambda+r_{t} d_{t}\right)=0 \quad \text { if slip }\left(\left\|\Lambda+r_{t} d_{t}\right\|>1\right) .\end{array}\right.$,

5. In the formulation proposed by Ben Dhia-Zarroug [11], we can introduce six regularization parameters to increase the control of the robustness. Indeed, let us consider $r_{n}^{S_{c}}, r_{t}^{S_{f}} \Rightarrow$ $S_{c}\left(\lambda_{n}-r_{n}^{S_{c}} d_{n}\right), S_{f}\left(\Lambda+r_{t}^{S_{c}} d_{t}\right)$ for the control of contact status. $r_{n}^{\hat{\lambda}_{n}}, r_{t}^{\lambda_{t}} \Rightarrow\left(\lambda_{n}-r_{n}^{\hat{\lambda}_{n}} d_{n}\right),\left(\Lambda+r_{t}^{\lambda_{t}} d_{t}\right)$ for the control of the augmentations. $r_{n}^{\text {Signo }}, r_{t}^{\text {Coul }} \Rightarrow$ $\left\{r_{\bullet}^{\bullet}\left(\lambda-S_{\bullet} d_{\bullet}\right)=0\right\}$ for the control of SignoriniCoulom's law.

Finally, the principle of virtual work consists to find $\left(u^{1}, u^{2}, \lambda_{n}, \Lambda\right)^{T}$ such that

$\{F\}\left(u^{i}, \lambda_{n}, \Lambda\right)=\left\{\begin{array}{l}\left\{F^{\text {int }}-F^{\text {ext }}\right\}+F^{\text {cont }}+F^{\text {frott }}=0 \text { (Weak form of the equilibrium) }, \\ \widetilde{F}^{\text {cont }}=0 \quad \text { (Weak form of Signorini) }, \\ \widetilde{F}^{\text {frott }}=0 \text { (Weak form of Coulomb) }\end{array}\right.$

In the following, we shall omit the arguments of $S_{\mathcal{O}} S_{f}$

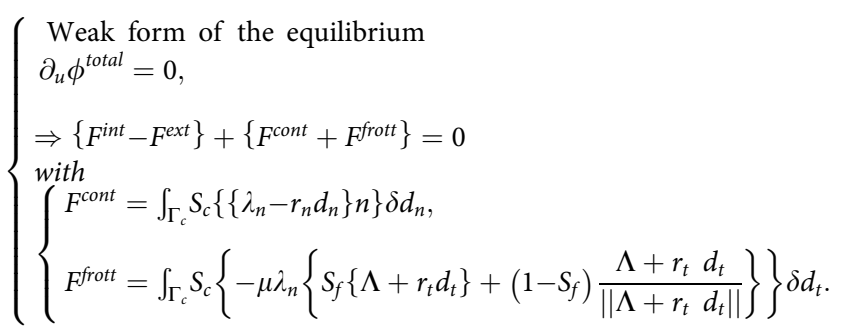




$$
\begin{gathered}
\left\{\begin{array}{l}
\text { Weak form of Signorini law } \\
\partial_{\lambda_{n}} \phi^{\text {cont }}=0, \\
\Rightarrow-\frac{1}{r_{n}} \int_{\Gamma_{c}}\left\{\lambda_{n}-S_{c}\left\{\lambda_{n}-r_{n} d_{n}\right\}\right\} \delta \lambda_{n}=0 .
\end{array}\right. \\
\left\{\begin{array}{l}
\text { Weak form of Coulomb law } \\
\partial_{\Lambda} \phi_{C\left(\lambda_{n}\right)}^{\text {fric }}=0, \\
\Rightarrow \widetilde{F}^{\text {frott }}=\frac{1}{r_{t}} \int_{\Gamma_{c}} \mu \lambda_{n} S_{c} \Lambda \delta \Lambda-\frac{1}{r_{t}} \int_{\Gamma_{c}} \mu \lambda_{n} S_{c} S_{f}\left(\Lambda+r_{t} d_{t}\right) \delta \Lambda, \\
-\frac{1}{r_{t}} \int_{\Gamma_{c}} \mu \lambda_{n} S_{c}\left(1-S_{f}\right) \frac{\Lambda+r_{t} d_{t}}{\left\|\Lambda+r_{t} d_{t}\right\|} \delta \Lambda+\int_{\Gamma_{c}}\left(1-S_{c}\right) \Lambda \delta \Lambda .
\end{array}\right.
\end{gathered}
$$

Let us now expose the discretization aspects of the formulation.

\subsection{Discretization aspects}

This work suits to the classical node-to-segment or point-to-segment contact elements. Point-to-segment contact uses gauss integration points to ensure that the contact law is strictly verified in a weak form. Various quadrature techniques for point-to-segment elements are implemented: Gauss, Newton-Cotes, Simpson. The space of approximations is precised below following the isoparametric concept.

\subsubsection{Approximation of unknowns}

$$
\begin{aligned}
& \text { Standardunknowns } \\
& X^{i, h}=\left[N^{i}\right]\left\{\chi^{i}\right\}, \\
& x^{i, h}=\left[N^{i}\right]\left\{\mathrm{Y}^{i}\right\}, \\
& u^{i, h}=\left[N^{i}\right]\left\{U^{i}\right\} .
\end{aligned}
$$

$$
\begin{aligned}
& \text { Contactunknowns } \\
& d^{i, h}=\left[\begin{array}{ll}
\Phi^{s} & -\Phi^{\text {mait }}
\end{array}\right] \quad\left\{\begin{array}{ll}
U^{s} & U^{\text {mait }}
\end{array}\right\}^{\mathrm{T}}, \\
& \lambda_{n}^{h}=\left[\Phi^{s}\right] \quad\left\{L_{\lambda}^{s}\right\}^{\mathrm{T}}, \\
& \Lambda_{\alpha}^{h}=\left[\Phi^{s}\right] \quad\left\{L_{\Lambda_{\alpha}}^{s}\right\}^{\mathrm{T}}, \quad \alpha=1,2,
\end{aligned}
$$

where $\chi^{i}, Y^{i}$, and $U^{i}$ represent, respectively, the nodal initial coordinates, the nodal current coordinates and the nodal displacements, $d^{i, h}$ represents the approximated gap vector in the global coordinate, $L_{\lambda}^{s}, L_{\Lambda_{\alpha}}^{s}$ represent the normal and tangential values of contact pressure in the global coordinate. They are assimilated to the Lagrange multipliers, $\left[N^{i}\right]$ are the classical Lagrange shape functions in the interior of the bodies, and $\Phi^{s}, \Phi^{m}$ are the shape functions on the contact surface. They are the trace functions of $N^{i}$

\subsubsection{Approximation of the local contact framework}

The local contact framework is the first parameter to handle. The discrete tangential and normal vectors in a point of the master surface are computed as follows:

$$
\left\{\begin{array}{l}
t^{m, \alpha, h}=\left[\partial_{\zeta_{\alpha}} \Phi^{m, h}\right]\left\{\mathrm{Y}^{m}\right\}, \quad \alpha=1,2, \\
n^{m, h}=-\frac{t^{1, h} \wedge t^{2, h}}{\left\|t^{1, h} \wedge t^{2, h}\right\|} .
\end{array}\right.
$$

\subsubsection{Approximation of level-set functions}

Level-set functions $\left(S_{c}, S_{f}\right)$ are nonsmooth operators which need specific techniques for their approximation. A collocation method is retained. For example, consider in Figure 2 a quadratic elementary surface with extremities (1) and (2). This elementary surface has a collection of contact points which is assimilated to the integration points (a) and (b). The level-set functions are first evaluated in contact points, then each contact point generates a new contact element with constant level-set:

1. First step: Evaluation of the level-set function on each contact point

$S_{c}\left(x^{i}\right)=S_{c}\left(\lambda_{n}^{h}\left(x^{i}\right)-r_{n} d_{n}^{h}\left(x^{i}\right)\right)= \begin{cases}1 & \text { if } \lambda_{n}^{h}\left(x^{i}\right)-r_{n} d_{n}^{h}\left(x^{i}\right) \leq 0, \\ 0 & \text { otherwise },\end{cases}$

where $x^{i}$ are the contact points on the elementary contact surfaces.

2. Second step: Approximation of the level-set function in the surface element

$$
S_{c}(x)=\delta\left(x-x^{i}\right) S_{c}\left(x^{i}\right), \quad x \in \Gamma_{c}^{e}\left(x^{i}\right)
$$

with $\Gamma_{c}^{e}\left(x^{i}\right)$ being the elementary node-to-segment contact surface generated by a contact point $x^{i}$ and Dirac shape function:

$$
\delta\left(x-x^{i}\right)= \begin{cases}1 & \text { if } \quad x \in \Gamma_{c}\left(x^{i}\right), \\ 0 & \text { otherwise. }\end{cases}
$$




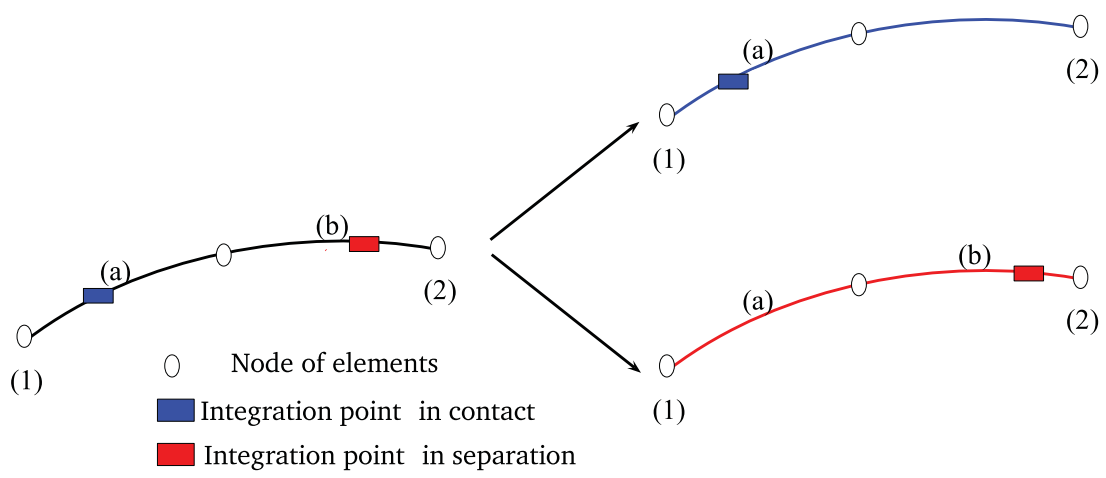

Figure 2. Collocation method for level-set functions (a) Geometry variation: left FPM, right GNM, (b) Coulomb's threshold variation: left FPM, right GNM and (c) Contact status variation: left FPM, right GNM.

In other words, we use a collection of contact points situated on a physical elementary surface which dictate the frictional-contact status of any point on the contact element.

\subsubsection{Remark: Numerical integrations}

It is kept in mind, the fact that the point-to-segment contact elements used can lead to incompatibilities (or oscillations) due to the nonconforming mesh at the interface of contact. In this case, specific quadrature strategies are used. They are reduced integration method or P2-P1 contact elements. The problem of oscillations can also be due to the discontinuity of normals as it was shown in [23]. To come over this difficulty, isogeometric contact elements based on NURBS or Hermite basis function can be performed to remove the problem. But in this article, we only use a basic strategy based on a moyennation of the normals of neighbor master segments.

\section{Solutions techniques}

\subsection{Linearization technique}

Let us denote by

$$
X^{h}=\left(U^{h}, \lambda_{n}^{h}, \Lambda^{h}\right) \text { the global unknowns vector }
$$

$\left\{F^{h}\left(X^{h}, p\left(X^{h}\right)\right)\right\}=\partial \phi^{\text {tot } h}\left(X^{h}, p\left(X^{h}\right)\right)$ the algebraic system to solve

$p\left(X^{h}\right)$ is the parameter of the system. These parameters are:

1. local contact frameworks and node-to-segment elements,

2. friction threshold associated with a contact point, and

3. frictional contact status of a point.
The algebraic system can be simplified by performing a fixed-point strategy on parameters:

$\left\{\right.$ Step 1: Find the fix point value $p^{0}: p\left(X^{h}\right)=p^{0}$,

Step2: Find the equilibrium state $X^{h}$ by Newton method which solves $F^{h}\left(X^{h}, p^{0}\right)=0$.

In the Newton method, we use a first-order Taylor's expansion of system (20):

Find $\Delta X^{h}$ such that

$$
\left[K^{\text {tot }, h}\left(X^{h}, p^{0}\right)\right] \Delta X^{h}+F^{h}\left(X^{h}, p^{0}\right)+O\left(X^{h}\right) \approx 0
$$

with $\left[K^{t o t, h}\left(X^{h}, p^{0}\right)\right]=\Delta F^{h}\left(X^{h}, p^{0}\right)$ being the global discrete matrix.

$$
\begin{gathered}
{\left[K^{\text {tot }, h}\left(X^{h}, p^{0}\right)\right]=\left[K_{\text {material }}^{\text {int, }}\left(U^{h}\right)\right]+\left[K_{\text {geometric }}^{\text {int, } h}\left(U^{h}\right)\right]} \\
+\left[K_{\text {contact }+ \text { friction }}^{\text {int, }}\left(X^{h}, p^{0}\right)\right] .
\end{gathered}
$$

\subsection{Fixed-point method}

In this method, most of the frictional-contact non linearities are simplified by fixed-point loop. The most external loop is on geometry.

\subsubsection{Fixed-point loop on geometry}

NTS elements depend on the geometric configuration during the computation. Then, one can reduce the complexity of the problem by fixing the NTS elements: a slave point is always coupled to the same master segment no matter what the intensity of the deformation process. During the Newton loop, the equilibrium configuration and the local contact framework are updated. A natural criterion to evaluate the convergence on geometry is based on displacements of the bodies (see Figure 3a). 


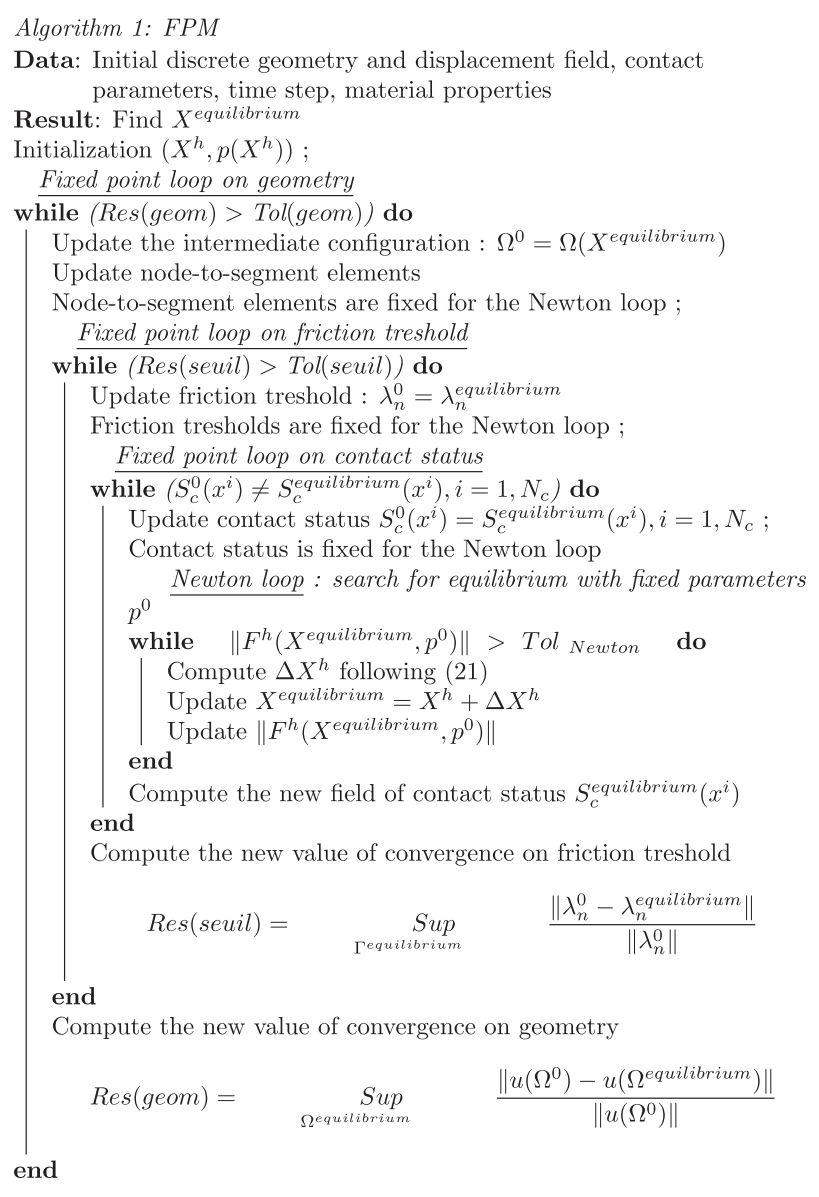

The next loop is more classical and it concerns friction threshold.

\subsubsection{Fixed-point loop on friction threshold}

Coulomb's friction threshold depends on contact pressure which is not known a priori. Then, a sequence of Tresca's friction law is used to approximate the Coulomb's one (see Figure 3b).

Data: Initial discrete geometry and displacement field, contact parameters, time step, material properties

Result: Find $X^{\text {equilibrium }}$

Initialization $\left(X^{h}, p\left(X^{h}\right)_{0}\right.$;

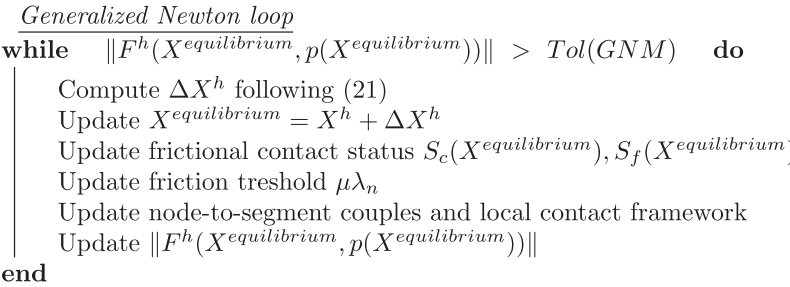

\subsubsection{Fixed-point loop on contact status}

Level-set functions are nonsmooth operators which can induce the lack of convergence of the Newton loop due to "cycling effects": the contact status of a pathological point does change permanently during the Newton loop. To increase the regularity of the discrete operators in the Newton loop, a fixed-point strategy is used only on contact status (see Figure 3c).

\subsubsection{Consequence on linearization}

1. The Fixed-point loop on friction threshold leads to symmetrized frictional contact matrix $\left[K_{\text {contact }+ \text { friction }}^{h}\right]$. Indeed, $\Delta \lambda_{n}^{h}=0 \Rightarrow K_{c f}^{\Lambda \lambda_{n}}=0$

2. In large deformations process, the local contact framework is updated then

$$
\Delta \delta d_{n} \neq 0, \quad \Delta \delta d_{t} \neq 0 .
$$

In small deformations process, the local contact framework is not updated

$$
\Delta \delta d_{n}=0, \quad \Delta \delta d_{t}=0 .
$$

In this work, a combination of these two concepts are implemented no matter what the deformation process: the local contact framework is updated at each Newton iteration but the second variation of kinematical quantities is not computed. It leads to a "not consistent" linearization of the discrete operators, then the Newton loop is a quasiNewton loop. But we will omit this fact in what follows.

3. The convergence of the Newton loop is

$$
\left\|F^{h}\left(X^{\text {equilibrium }}, p^{0}\right)\right\|=\frac{\left\|F^{\text {int }}+F^{\text {cont }+ \text { fric }}-F^{\text {ext }}\right\|}{\left\|F^{\text {ext }}\right\|} .
$$

\subsection{Generalized Newton method (GNM)}

Data: Initial discrete geometry and displacement field, contact parameters, time step, material properties

Result: Find $X^{\text {equilibrium }}$

Initialization $\left(X^{h}, \Omega^{0}\right)$;

Fixed point loop on geometry

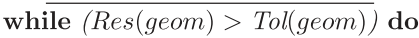

Update the intermediate configuration : $\Omega^{0}=\Omega\left(X^{\text {equilibrium }}\right)$

Update node-to-segment elements

Node-to-segment elements are fixed for the Newton loop

Partial Newton loop

while $\left\|F^{h}\left(X^{\text {equilibrium }}, \Omega^{0}\right)\right\|>$ Tol $_{P N M}$ do

Compute $\Delta X^{h}$ following (21)

Update $X^{\text {equilibrium }}=X^{h}+\Delta X^{h}$

Update frictional contact status

$S_{c}\left(X^{\text {equilibrium }}\right), S_{f}\left(X^{\text {equilibrium }}\right)$

Update friction treshold $\mu \lambda_{n}$

Update $\left\|F^{h}\left(X^{\text {equilibrium }}, \Omega^{0}\right)\right\|$

end

Compute the new value of convergence on geometry

$$
\operatorname{Res}(\text { geom })=\quad \operatorname{Sup}_{\Omega^{\text {equilibrium }}} \quad \frac{\left\|u\left(\Omega^{0}\right)-u\left(\Omega^{\text {equilibrium }}\right)\right\|}{\left\|u\left(\Omega^{0}\right)\right\|}
$$

end

In this method, all contact parameters change during the Newton iterations. 

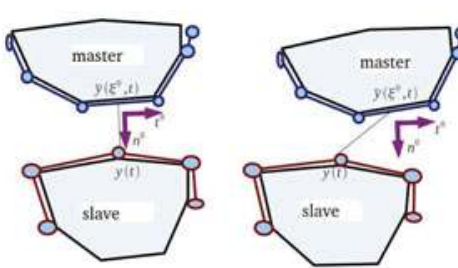

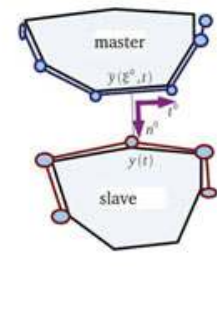

Itération : N

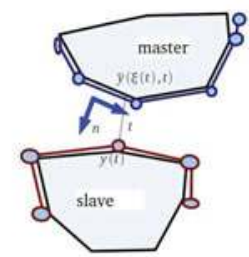

Itération : $\mathrm{N}+1$

(a) Geometry variation: left FPM, right GNM
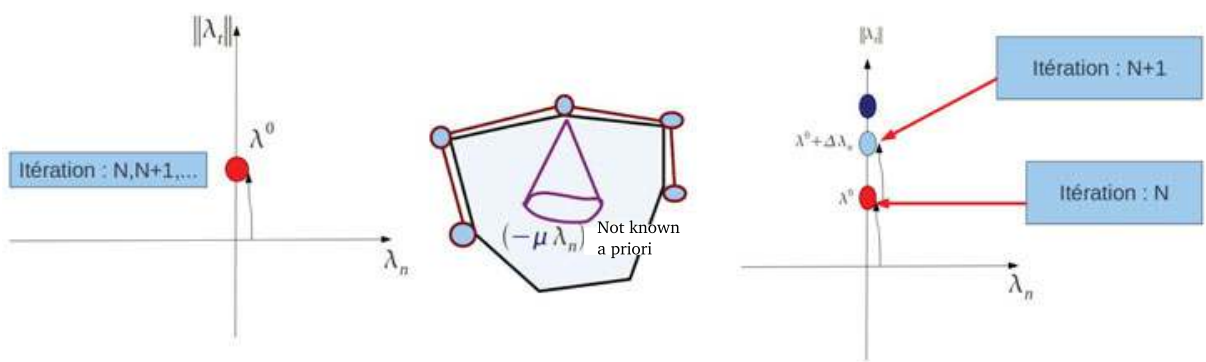

(b) Coulomb's treshold variation: left FPM, right GNM
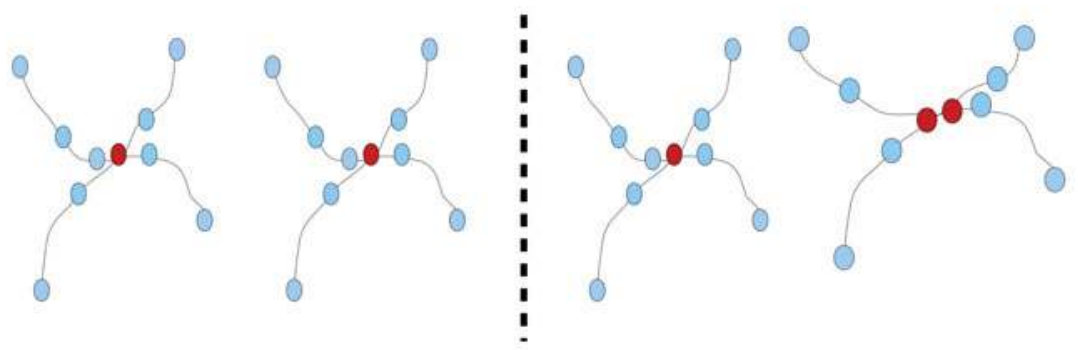

(c) Contact status variation: left FPM, right GNM

Figure 3. Comparison of FPM and GNM.
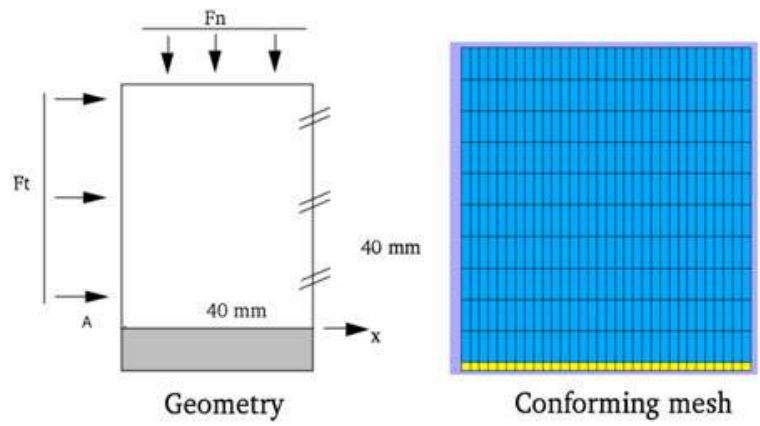

Figure 4. Elementary test of a compressed square plate.

\subsubsection{Consequence on linearization}

1. The variation of friction threshold has its influence on tangential motion: $\Delta \lambda_{n}^{h} \neq 0 \Rightarrow K_{c f}^{\Lambda \lambda_{n}} \neq 0$. The asymmetrical character of the frictional contact matrix [ $\left.K_{\text {contact }+ \text { friction }}^{h}\right]$ is physically interpreted as follows: friction depends on contact pressure but the reverse is not true.

2. It is implemented a consistent linearization of the discrete system. 

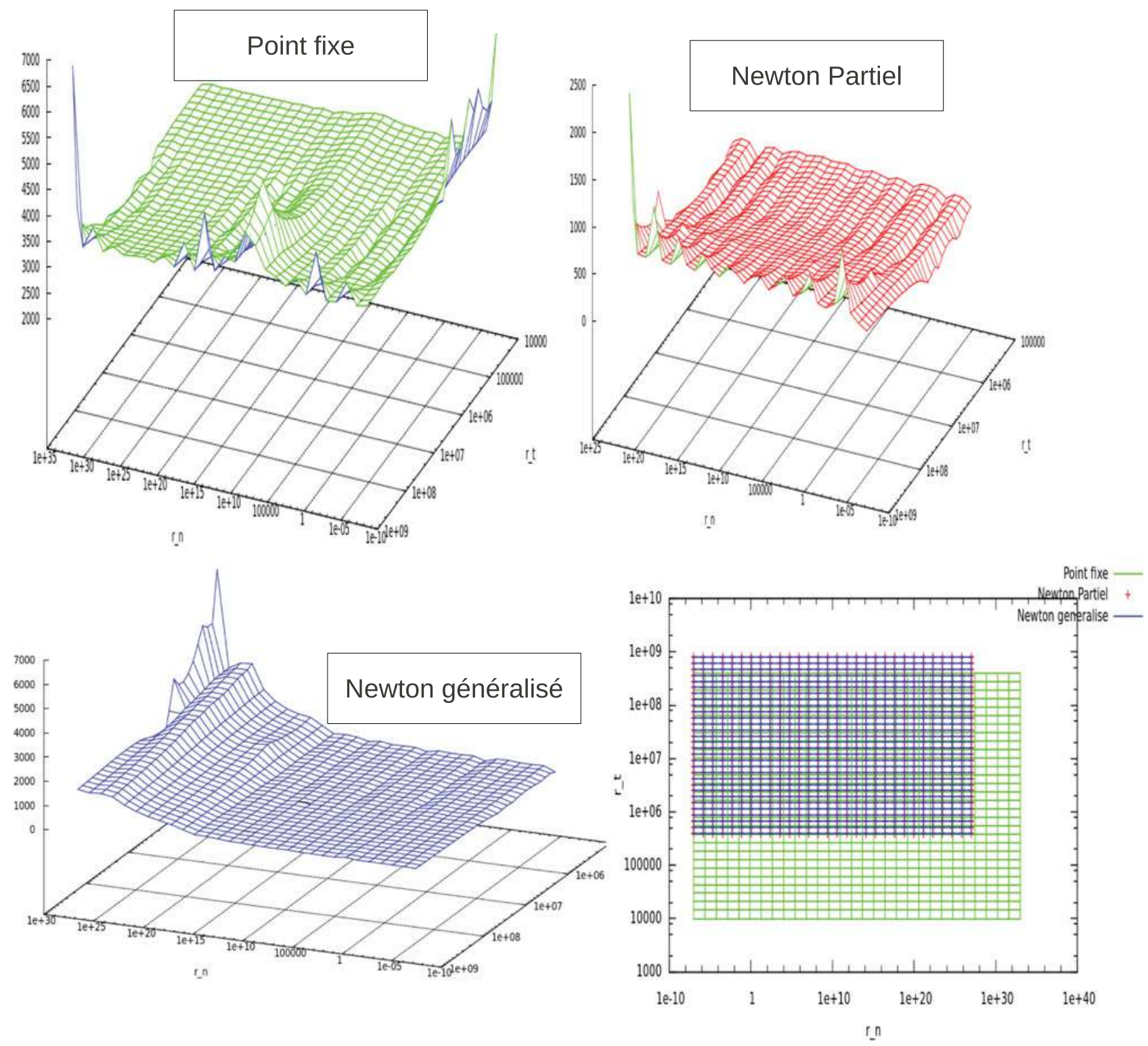

Figure 5. Range of values of $r n, r t$ : empiricalobservation.

3. It is built in equivalent criteria of convergence as in the FPM:

$$
\begin{gathered}
\left\|F^{h}\left(X^{\text {equilibrium }}, p^{\text {equilibrium }}\right)\right\|=\frac{\left\|F^{\text {int }, k+1}+F^{\text {cont }+ \text { fric }, k+1}-F^{\text {ext }, k+1}\right\|}{\left\|F^{\text {ext }, k+1}\right\|} \\
+\operatorname{Sup}_{\Gamma^{\text {cequilibrium }}} \frac{\left\|\lambda_{n}^{k+1}-\lambda_{n}^{k}\right\|}{\left\|\lambda_{n}^{k+1}\right\|}, \\
+\operatorname{Sup}_{\Omega^{\text {equilibrium }}} \frac{\left\|u\left(\Omega^{k+1}\right)-u\left(\Omega^{k}\right)\right\|}{\left\|u\left(\Omega^{k+1}\right)\right\|}
\end{gathered}
$$

where $k+1$ is the current Newton iteration. These criteria insure the convergence on the equilibrium and on the contact parameters.

\subsection{Partial newton method}

Partial Newton method (PNM) is a basic idea of a hybridation of GNM and FPM based on heuristic observations. In the GNM, detection of contact pairs and the variations of kinematics values have to be computed at each Newton iteration. This is especially true for large deformations, large slips, and deformable-to-deformable transformations. The change of the configuration produces at each iteration a new system to solve. This fact can induce the lack of convergence since we have to check the fact that a contact pairing has not changed during the iteration. Furthermore, GNM can be seen like a global predictor-corrector 
algorithm for the contact laws. Indeed, Coulomb's law is nonassociated so it is used a similar technique of "return-mapping for plasticity" to ensure the satisfaction of the friction law. In other hand, the fixed-point loop allows a friendly system to solve at each Newton iteration: in the case of frictionless and elasticity behavior, the problem is smooth enough to obtain convergence. But the drawback is the fact that it needs, in complex cases, many iterations on fixedpoint loop. For example, suppose that for a largescale problem of $120,000 d^{\prime} f^{\prime} s$, FPM needs four iterations for the geometry loop, five on the friction one, eight on the contact loop, and five for the Newton loop then the total iterations of Newton is 800. According to this example, FPM is prohibited since the factorization of the system and the computation of the material tangent matrix take a lot of time.

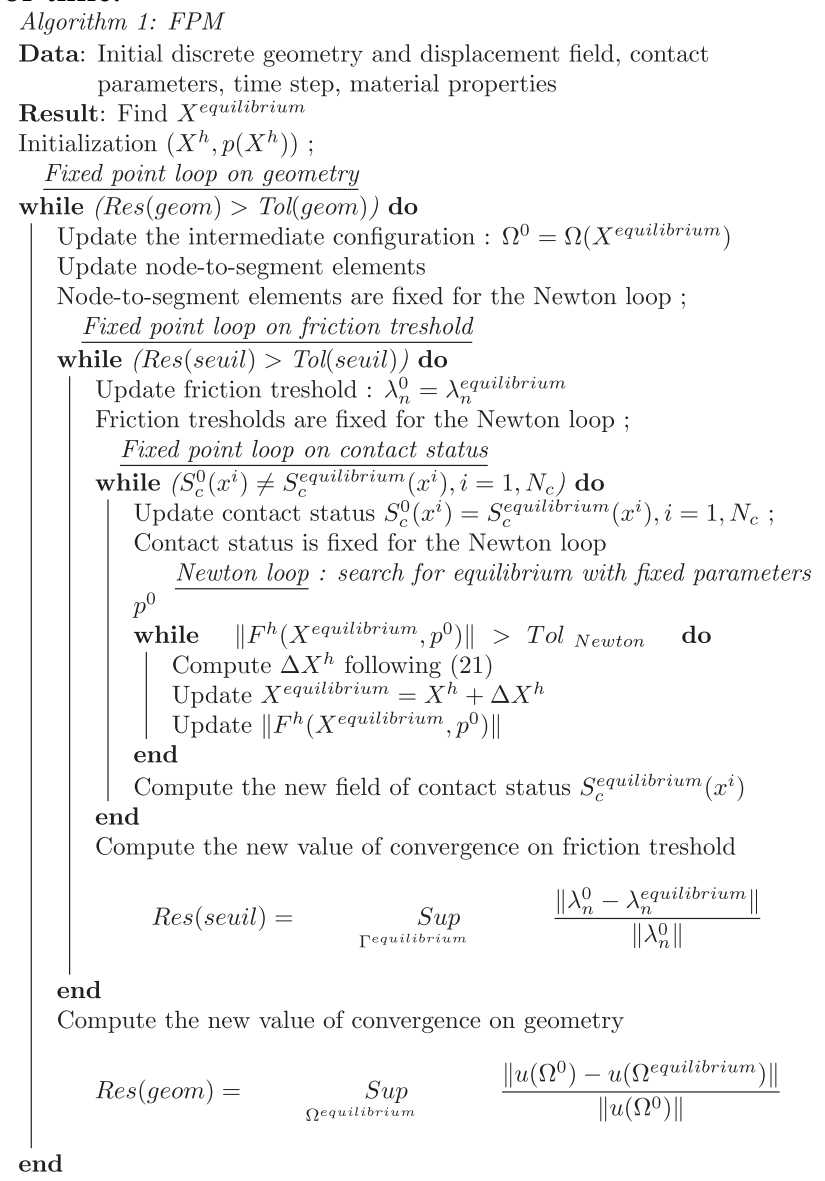

Remark Many variants of PNM can be implemented. For example, instead of the fixed-point loop on the geometry one can use the fixed-point loop on the friction threshold in the PNM. We present in this article, the most efficient PNM algorithm (fixed-point loop on the geometry).

The next section concerns show the behavior of GNM, PNM, and FPM on various numerical examples.

\section{Numerical tests}

\subsection{A simple $2 D$ case: influence of regularization parameters}

A square plate is compressed on an indeformable plate body. The material properties of the square plate are: $\nu=0.2, E=1.310^{5} \mathrm{~N} / \mathrm{mm}^{2}$. The boundary condition is such that the square plate is clamped on its right side and compressed by a normal and tangential force (see Figure 4). This example shows that the range of values of $r_{n}, r_{t}$ allowed to ensure the convergence is not the same according to FPM, PNM, and GNM see Figure 5. This is especially true when soft materials are involved. That is the reason why an empirical choice on the upper bound of the numerical parameters is used:

$$
r_{n} \leq 100 \min \left\{E^{i}\right\}, \quad r_{t} \leq 10 \min \left\{\mu E^{i}\right\}
$$

with $i=1,2 . . N$ stands for contacting bodies

and $E^{i}$ is the elastic parameter of the body

\subsection{Ring on block test}

\subsubsection{Description of the problem}

This example is similar to the one presented in [24]. A soft composite ring composed by two bonded circular rings (inner ring and outer ring) is submitted to large deformation process, frictional contact and large slip. The ring will contact a rectangular soft block (see Figure 6a).

A vertical displacement is imposed at the top of the ring $u_{y}=90 \mathrm{~mm}$ and the bottom of the block is clamped. The mesh is composed of linear triangular and hexaedral elements.

In this problem, drastic changes occur on active sets, NTS elements, and friction threshold. We show the influence of the friction coefficients on the convergence of the algorithms.

The elastic properties are

\subsubsection{Influence of friction coefficient on algorithms}

The aim of this subsection is to show the performance of each algorithm for different values of the friction coefficient in the elastic material behavior case. The results are summarized in the table below.

\begin{tabular}{lccc}
\hline Materials & TRIA3 & HEXA4 & SEG2 \\
\hline inner ring & 723 & & \\
outer ring & 667 & & \\
block & & 2500 & 79 \\
slave surface (outer ring) & & & 50 \\
master surface (block) & & & \\
\hline
\end{tabular}




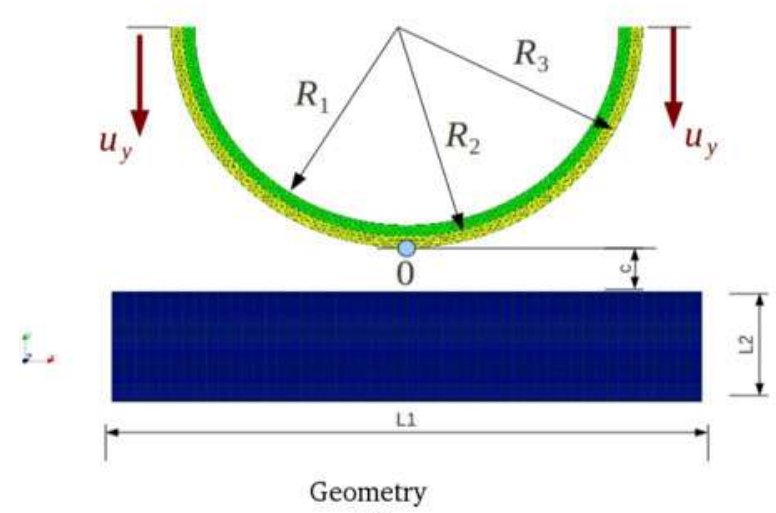

(a)
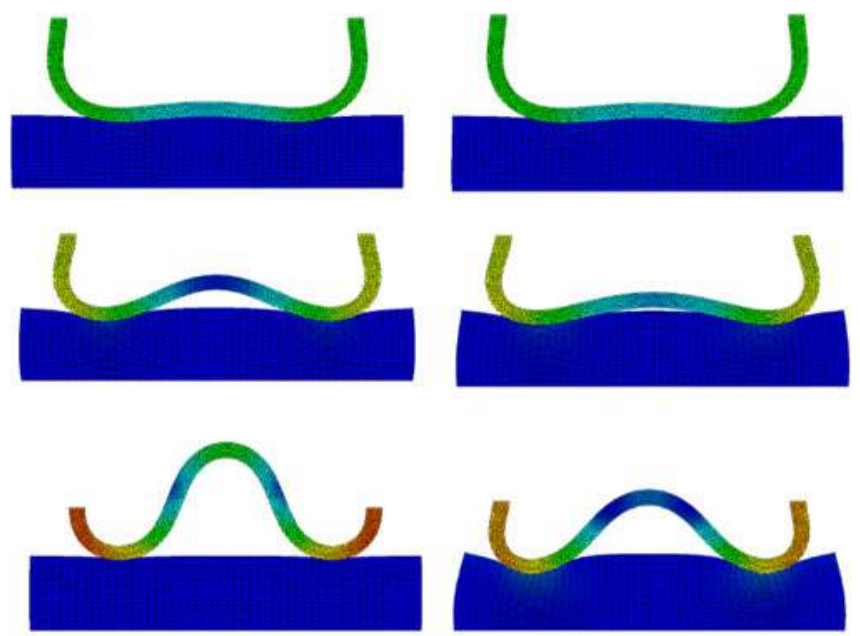

Displacement
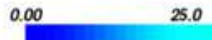

50.0

(b)

Figure 6. Ring on block test: (a) Description of the geometry, (b) On left: frictionless cases, on right: with friction $\mu=0.3$.

\begin{tabular}{lcc}
\hline Materials & Young modulus $(\mathrm{MPa})$ & Poisson ratio \\
\hline inner ring & $10^{5}$ & 0.3 \\
outer ring & $10^{3}$ & 0.3 \\
block & 3. $10^{2}$ & 0.3 \\
\hline
\end{tabular}

We can see that FPM is performant in the case of $\mu=0.5$ than the case of $\mu=0.3$. It is due to the fact that contact elements are "glued" because of Coulomb's threshold so that Geometry and contact status loops can be neglected. GNM fails to converge in the case of $\mu=0.5$ because a small variation of parameters can induce a "dramatic convergence" due to the fact that all nonlinearities are solved in one loop of iterations. PNM seems to be robust in all cases and it is performant in the case of $\mu=0.3$. This case will be discussed in Section 16. Indeed, we can consider three limits situations: (1) adherence situation is preponderant, (2) slip situation is preponderant, (3) these two situations co-exist. One of the main contribution of this article is a discussion on an optimal choice of a solution technique depending on these three situations. GNM is affected by the numerical parameters of augmentations as depicted in some works in the literature [19-21].

\subsection{Three-dimensional ironing test}

\subsubsection{Description}

We consider now a three-dimensional test as sketched in Figure 4.3a. A deformable rectangular block is crushed by a rigid cylinder block.
Geometrical sets: $\quad R_{1}=26.7 \mathrm{~mm}, \quad e=$ $2.1 \mathrm{~mm}, \quad L_{1}=90 \mathrm{~mm}, \quad L_{2}=40 \mathrm{~mm}, \quad l=30 \mathrm{~mm}$.

Mesh sets: The slave rectangular block is modeled by 360 hexaedra elements (HEXA8) with 150 potentially in contact. We use the linear shape functions. The master cylinder block is modeled by 220 HEXA8.

Material behaviors: We suppose that the rectangular block can follow three behaviors: elasticity with Green-Lagrange transformations, elastic-plasticity with Simo-Miehe decomposition of the deformation [25] and finally hyper-elastic with Green-Lagrange transformations.

a. Elastic behavior: $E=1 \mathrm{MPa}, \quad \nu=0.3$.

b. Elastic-plastic behavior: $E=1 M P a, \nu=$ $0.3, \sigma_{\text {Yield }}=0.3 \mathrm{MPa}, \quad H_{\text {hardening }}=0.6 \mathrm{MPa}, \quad E=$ $E^{e} E^{p}$

with $E^{e}$ being the elastic part of the deformation and $E^{p}$ the plastic part.
c. Hyper-elastic behavior: $C_{10}=1 \mathrm{MPa}$, $C_{01}=0.0 \mathrm{MPa}, \quad C_{20}=0.0 \mathrm{MPa}, \quad \nu=0.3$.

Algorithm sets: We use FPM, GNM, and PNM. Additionally, we compare these algorithms to a typical constrained optimization method named: Gradient Conjugate and Projected method [9] (GCP).

Numerical simulations: The indentation phase is simulated in $1 s$ in 10-time steps. The final position of the cylinder is $U_{z}(1 s)=-14 \mathrm{~mm}$. This phase is 


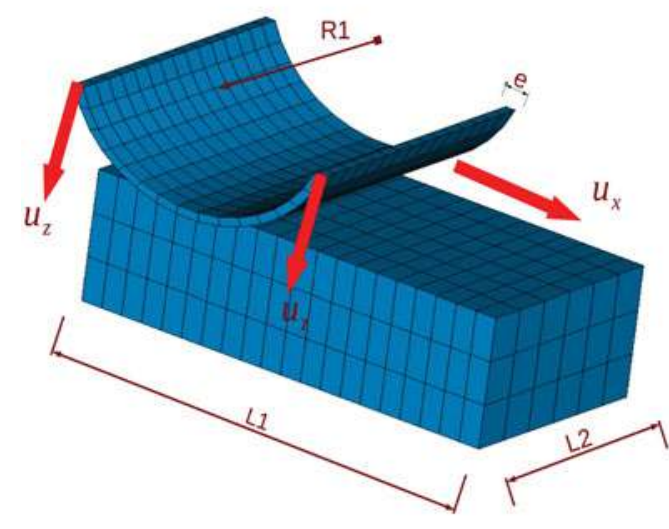

(a)

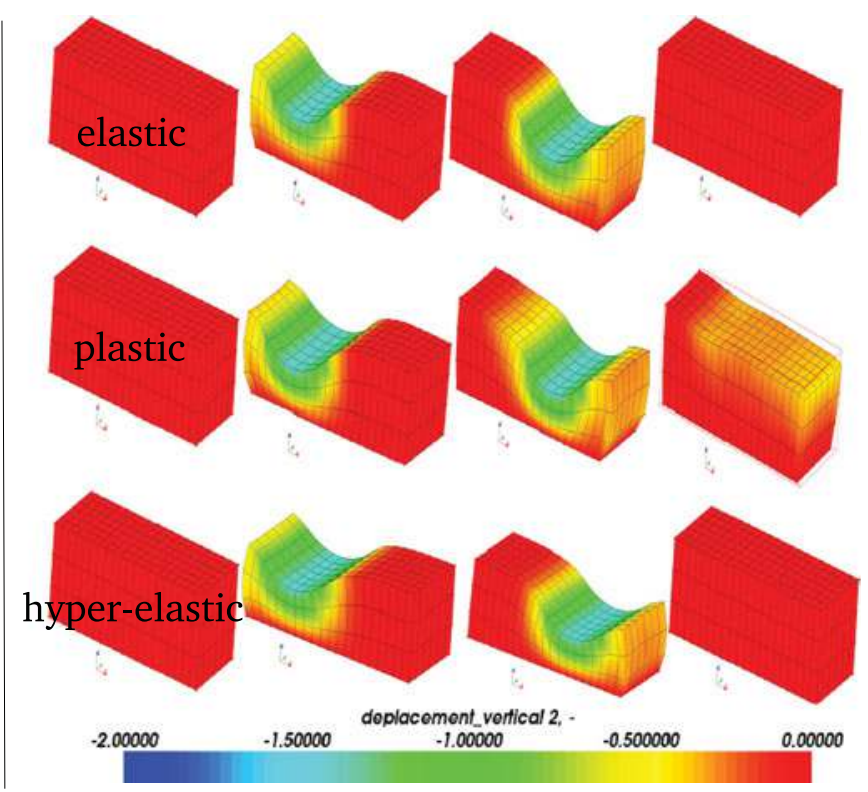

(b)

Figure 7. 3D ironing test: (a) Geometry, (b) deformed shape in elastic, plasticity, and hyper-elastic cases.

\begin{tabular}{lccc}
\hline FPM & $\begin{array}{c}\text { Number of } \\
\text { time steps }\end{array}$ & $\begin{array}{c}\text { Total Newton } \\
\text { iterations }\end{array}$ & $\begin{array}{c}\text { NTS } \\
\text { reconstructions }\end{array}$ \\
\hline$\mu=0.0$ & 293 & 2110 & 1829 \\
$\mu=0.3$ & 260 & 7420 & 1296 \\
$\mu=0.5$ & 250 & 5040 & 1057 \\
PNM & $\begin{array}{c}\text { Number of } \\
\text { time steps }\end{array}$ & $\begin{array}{c}\text { Total Newton } \\
\text { iterations }\end{array}$ & NTS reconstructions \\
$\mu=0.0$ & 220 & 3763 & \\
$\mu=0.3$ & 310 & 5413 & 1829 \\
$\mu=0.5$ & 425 & 5287 & 1456 \\
GNM & Number of & Total Newton & NTS reconstructions \\
& time steps & iterations & \\
$\mu=0.0$ & 295 & 2403 & 2698 \\
$\mu=0.3$ & 351 & 4516 & 4700 \\
$\mu=0.5$ (failed) & $\times$ & $\times$ & $\times$ \\
\hline
\end{tabular}

followed by a slip motion of the cylinder $U x(2 s)=$ $90 \mathrm{~mm}$ in 90 -time steps.

\section{Results and interpretation}

Figure $4.3 \mathrm{~b}$ shows the deformation process of the structure according to the material behaviors. Note the fact that there exists a residual stress in the case of the elastic-plastic behavior. We compare the algorithms developed to a constrained optimization method so-called GCP-Gradient Conjugate and Projected method which we can see in [9] (Figure 7).

As it is shown in the statistic figure (see Figure 8), GCP algorithm is very performant when it converges but the drawback is the robustness. It fails to converge in the case of the plasticity (see Figure 9) or in the presence of the friction. The unconstrained

\begin{tabular}{lccc}
\hline$\mu=0$ & FPM & PNM & GNM \\
\hline Wall clock time (hours) & $35 \mathrm{~h}$ & $42 \mathrm{~h}$ & $8 \mathrm{~h}$ \\
Differences & Reference & $<1 \%$ & $<5 \%$ \\
$\mu=0.3$ & FPM & PNM & GNM \\
Wall clock time (hours) & $107 \mathrm{~h}$ & $45 \mathrm{~h}$ & Failed \\
Differences & Reference & $<3 \%$ & $\times$ \\
\hline
\end{tabular}

optimization methods developed in this work seems to be more robust. In terms of performance, PNM seems to be a good compromise between FPM and GNM (see Figure 8). In terms of reliability, all algorithms converge to the same result (see Figure 9).

\subsection{A large-scale problem: expansion of a tube of a steam water}

The industrial application concerns a metal forming process called tube expansion, which consists in increasing the diameter of tube of a steam generator tube (nuclear plants) by using rollers. As illustrated in Figure 10, the rollers are in contact with the tube, and they follow a radial translation and axial rotation in order to put the tube in contact with the tube sheet (or the plate).

- Geometrical characteristics

Tube sheet: $R_{1}=100 \mathrm{~mm}, \quad R_{2}=11.6 \mathrm{~mm}, \quad$ and $E=12 \mathrm{~mm}$.

Tube: $\quad R_{3}=11.27 \mathrm{~mm}, \quad R_{4}=$ $10 \mathrm{~mm}$, and $L=70 \mathrm{~mm}$.

Rollers are modeled by hollow cylinders: $R_{5}=2.8 \mathrm{~mm}$. 

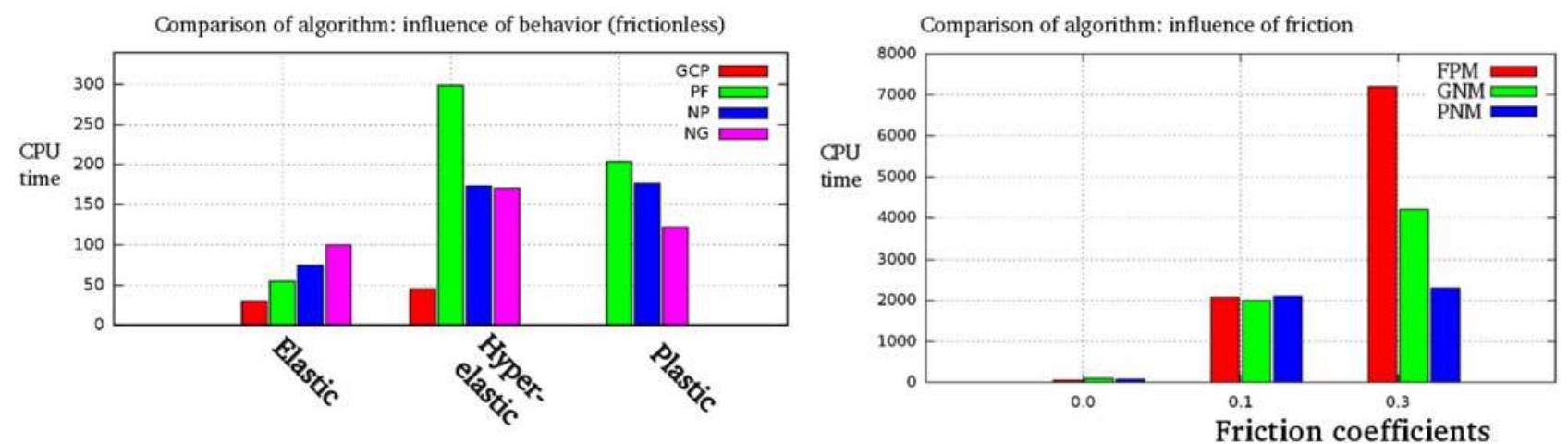

Figure 8. Statistic of performance.
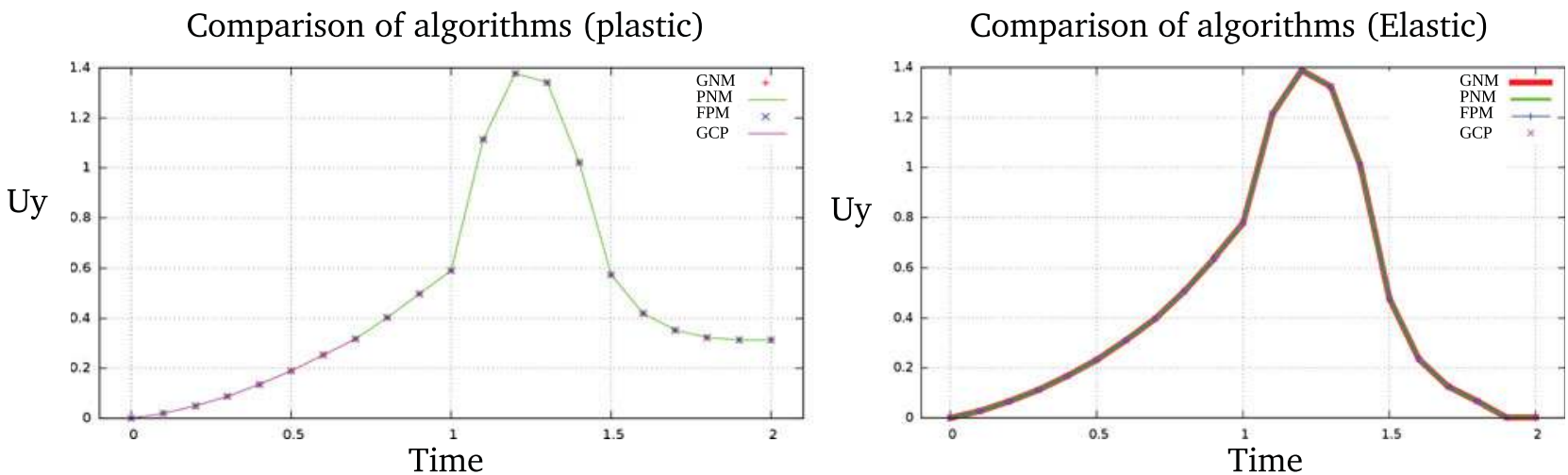

Figure 9. Vertical displacement of a central point in the slave surface $M(x=45 \mathrm{~mm}, y=20 \mathrm{~mm}, z=30 \mathrm{~mm})$ : (left $=$ plastic right $=$ elastic).


Figure 10. Large-scale problem. 


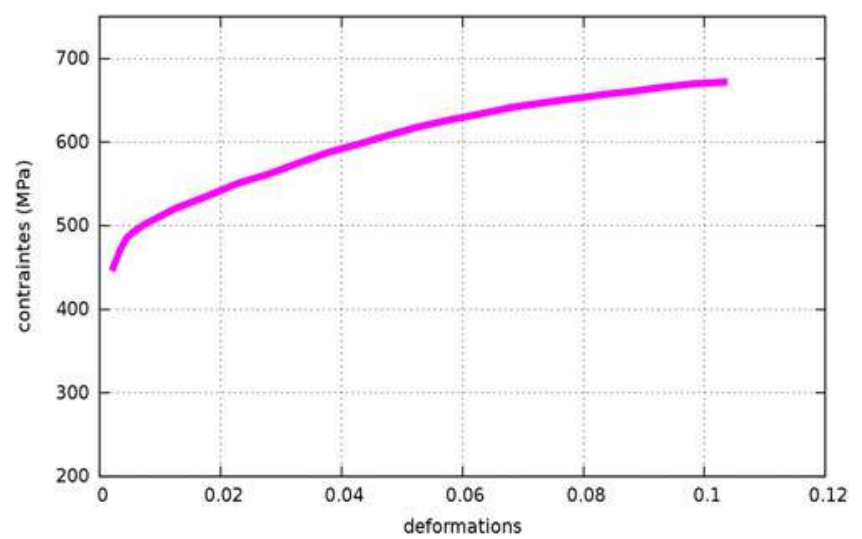

Figure 11 Expansion of tube: material behavior of the tube sheet.

- Mesh characteristics

We consider quadratic elements of HEXA27 (hexaedra with 27 nodes). The mesh is composed by 39612 nodes with 2000 slave nodes potentially in contact. There are contact zones between the interior of the tube and the rollers and the exterior of the tube and the tube sheet.

- Structural behavior

The tube sheet is modeled by a plastic material with a nonlinear and an isotropic hardening as illustrated in Figure 11 with yield stress: $\sigma_{Y}=$ $450 \mathrm{MPa}, E=204,000 \mathrm{MPa}$ The tube is modeled by a plastic material with a mixed and linear hardening rule of Prager type: $E=179,000 \mathrm{MPa}, \quad \sigma_{Y}=$ $335 \mathrm{MPa}, E_{t}=2726 \mathrm{MPa}, \quad$ and $E_{c}=1500 \mathrm{MPa}$ with $E_{t}$ being the isotropic hardening contribution and $E_{c}$ the kineamtic one (Prager constant).

- Simulation conditions

Initially, it exists a gap between the rollers and the tube. The rollers are submitted to spiral movement on $360 \mathrm{~s}$ with $1 \mathrm{~s}$ time step. The deformed shape and the equivalent distribution of the stress are proposed in Figure 12.

- Performance tests: comparison of FPM, PNM and GNM

To make an objective comparison of the algorithms, we perform this industrial problem in the sequential computation framework (single domain single thread).

The time of computation is due to large-scale problem with material nonlinearities (tube and tube sheet) and the presence of friction. Thanks to multi-threading implementation (single-domain multi threads), the time of integration of materials can be reduced significantly. Even in this case, the trend of the algorithms is still the same as in the sequential computation. This industrial application shows that PNM is the best algorithm to compute a frictional contact problem since it is more robust than GNM and more performant than FPM in frictional case.

\section{Discussion}

In this section, we summarize the behavior of the three types of algorithm developed in this work.

1. Frictionless case: Slip motion with zero (or negligible) tangential stress is preponderant. In this case, all the algorithms converge to the same solution. In our example, GNM seems to be faster than all other methods proposed. FPM is not performant because it must always check the fixedpoint loop on contact parameters no matter if the equilibrium was found or not.

In other complex cases, GNM can fail to converge due to contact cycling phenomena on some slave nodes.

This situation can be described as follows:

$$
\begin{array}{lr}
\text { Iteration }: k & \text { Iteration }: k+1, \\
\lambda_{n}^{k}-r_{n} d_{n}^{k}<0 & \lambda_{n}^{k+1}-r_{n} d_{n}^{k+1}>0 .
\end{array}
$$

The key idea to remove this situation is based on an optimal choice of the augmented numerical parameter such that

$$
\left(\lambda_{n}^{k}-r_{n}^{k+1} d_{n}^{k}\right)\left(\lambda_{n}^{k+1}-r_{n}^{k+1} d_{n}^{k+1}\right)>0 .
$$

2. Frictional case: Slip motion with non-negligible tangential stress is preponderant. Two limits situation can be considered: stick is preponderant (high-friction coefficients) or slip is preponderant (small friction coefficients). In the first case, GNM method seems to be a good strategy to perform the problem. In the second case, when slip with non-negligible tangential force is preponderant, GNM can involve many iterations due to drastic variation of contact parameters, especially the geometrical or friction. Then, PNM is a good technique to solve this kind of problems. All algorithms have their advantages and drawbacks. Let us precise that the conclusions above is not a general rule, it is simply some heuristics techniques used to perform large industrial scale problems in the context of sequential or multithreading or multidomains computational frameworks.

In GNM, it often appears cycling phenomena. Cycling effects can be due to: 


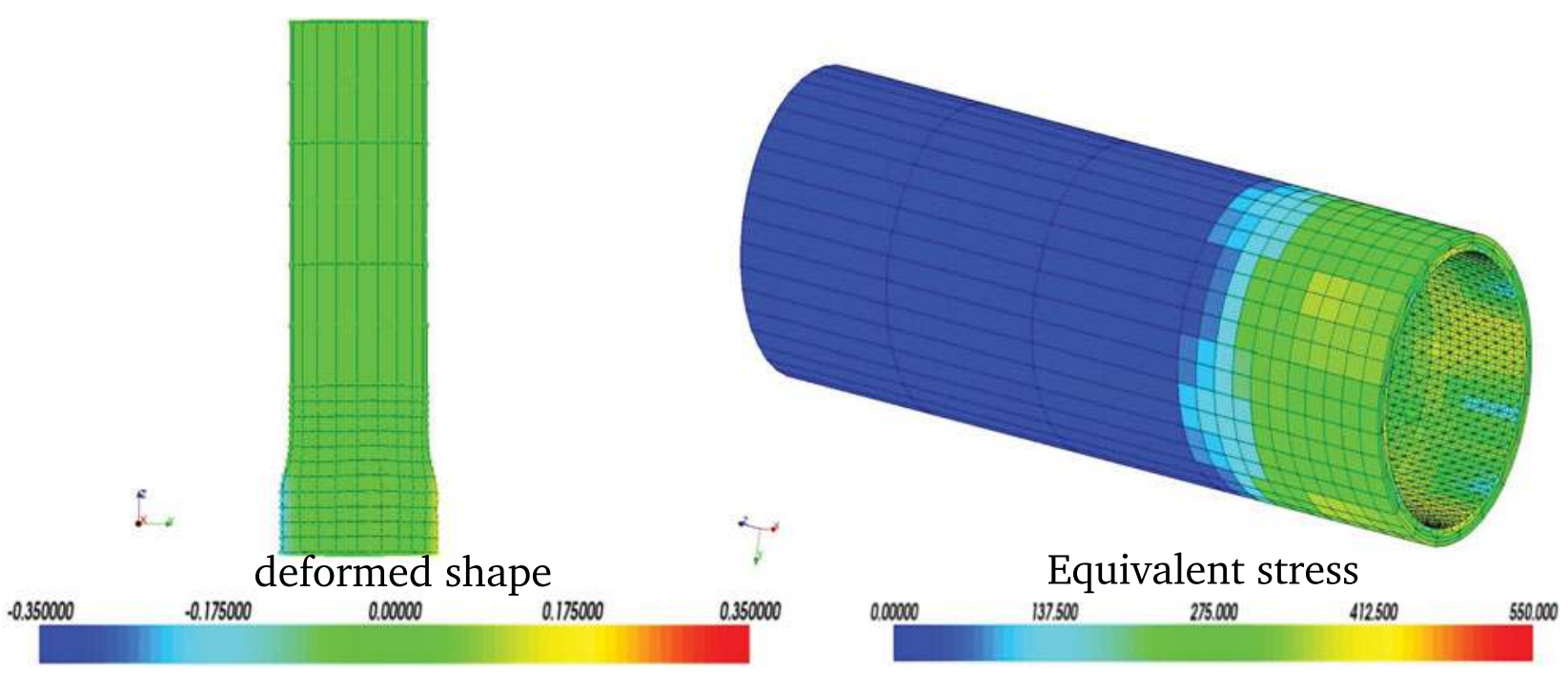

Figure 12. Large-scale problem.

- geometrical oscillations: a slave node oscillates between two masters segments. The solution consists to perform a technique of regularization of the master surface. Some smoothing techniques are described in $[7,21,23,26]$.

- frictional-contact status cycling: a couple of slave/ master always change its status due to the influence of the neighbors contact elements. These facts can be solved by choosing optimal augmented numerical parameters. The influence of the augmented numerical parameters is also exhibited in $[19,20]$. There exist two limits situations: backward/forward slip cycling and adherence/slip cycling. These facts can be described as follows:

Backward/forward slip cycling

$$
\begin{aligned}
& \left\{\begin{array}{l}
\text { Iteration k: backward slip } \\
\left\|\Lambda^{k-1}+r_{t} d_{t}^{k-1}\right\|>1 \\
\left\|\Lambda^{k}+r_{t} d_{t}^{k}\right\|>1 \\
\left(\Lambda^{k}+r_{t} d_{t}^{k}\right) \cdot\left(\Lambda^{k-1}+r_{t} d_{t}^{k-1}\right)<0
\end{array}\right. \\
& \qquad \begin{array}{l}
\text { Iteration } \mathrm{k}+1: \text { forward slip } \\
\left\|\Lambda^{k}+r_{t} d_{t}^{k}\right\|>1 \\
\left\|\Lambda^{k+1}+r_{t} d_{t}^{k+1}\right\|>1 \\
\left(\Lambda^{k+1}+r_{t} d_{t}^{k+1}\right) \cdot\left(\Lambda^{k}+r_{t} d_{t}^{k}\right)<0
\end{array}
\end{aligned}
$$

The key idea to solve backward/forward slip cycling is to fix some slave nodes to adherent status according to the fact that they are submitted to a very small slip motion.

Adherence/slip cycling $\left\{\begin{array}{l}\text { Iteration } \mathrm{k}: \text { adherence } \\ \left\|\Lambda^{k}+r_{t} d_{t}^{k}\right\|<1\end{array}\left\{\begin{array}{l}\text { Iteration } \mathrm{k}+1 \text { : slip } \\ \left\|\Lambda^{k+1}+r_{t} d_{t}^{k+1}\right\|>1\end{array}\right.\right.$.

The adaptation of $r_{t}$ is based on the fact that

$$
\left(1-\left\|\Lambda^{k}+r_{t}^{k+1} d_{t}^{k}\right\|\right)\left(\left\|\Lambda^{k+1}+r_{t}^{k+1} d_{t}^{k+1}\right\|\right)>0 .
$$

The FPM method is the most robust method due to the control of contact parameters so that cycling is avoid when finding equilibrium state. PNM combines the advantages of FPM and GNM.

\section{Conclusion}

In this article, we develop and compare three types of algorithms in order to treat frictional contact problem coupled with material nonlinearities. FPM is a solution technique based on minimization of the mechanical problem with some parameters under control and the GNM is a global predictor/corrector solution technique extended to frictional cases. These methods are tested in various physical frictional contact situations and we can see in this article their advantages and their inconvenient. To combine advantages of GNM and FPM, we develop the PNM method in the case of slip motion with non-negligible tangential stress. This last algorithm is adopted to treat large-scale industrial problems especially in nuclear plant.

Our future works will be devoted to a new algorithm with a heuristic technique to solve cycling contact phenomena. It will be explained an empirical 
choice of the upper bound of the augmented numerical parameter, a decomposition of the unilateral contact law and finally a specific treatment of the contact cycling.

\section{Acknowledgment}

Thanks to the main organizations which supported this work: Électricité De France R\&D, Laboratoire de Mécanique et d'Acustique CNRS and Code_Aster developpers team. Especially, we want to thank N. Tardieu, P. Massin, M. Guitton, M. Siavelis and G. Ferte for their fruitful contributions and their critical points of view.

\section{References}

[1] P. Chabrand, F. Dubois, and M. Raous, "Various numerical methods for solving unilateral contact problems with friction," Nonlinear Anal., vol. 28, no. 4-8, pp. 97-976, 1998.

[2] J. Haslinger, "Approximation of contact problems. Shape optimization in contact problems," in Book: Non-Smooth Mechanics and Applications, edited by J. J. Moreau and P. D. Panagiotopoulos, Wien - New York: Springer - verlag, vol. 302, 1988.

[3] F. Lebon, and M. Raous, "Multibody contact problem including friction in structure assembly," Comput Struct., vol. 43, no. 5, pp. 925, 1992.

[4] M. Raous, "Quasi-static Signorini problem with Coulomb friction and coupling to adhesion," in Book: New Developments in Contact Problems, edited by P. Wriggers and P. Panagiotopoulos, Wien - New York: Springer - verlag, vol. 384, pp. 101-178 1999.

[5] F. Kuss, and F. Lebon, "Stress based finite element methods for solving contact problems: Comparisons between various solution methods," Adv. Eng. Softw., vol. 40, no. 8, pp. 697-706, 2009.

[6] P. Bisegna, F. Lebon, and F. Maceri, "Relaxation procedures for soving signorini-Coulomb Contact problems," Adv. Eng., vol. 35, no. 10-11, pp. 595-600, 2004.

[7] Code_aster, Open source - general FEA software for analysis of structures and thermomechanics for studies and research. www.code-aster.org.

[8] S. Hueber, and B. I. Wohlmuth, "A primal-dual active set strategy for nonlinear multibody contact problems," Comp. Methods Appl. Mech Eng., pp. 27-29, 1995.

[9] M. Renouf and P. Alart, "Conjugate gradient type algorithms for frictional multi-contact problems: applications to granular materials," Comput. Methods Appl. Mech Eng., vol. 194, no. 18-20, pp. 2019-2041, 2005.

[10] P. Alart and A. Curnier, "A mixed formulation for frictional contact problems prone to newton like solution method," Comp. Methods Appl. Mech. Eng., vol. 92, no. 3, pp. 353-375, 1991. pages

[11] H. Ben Dhia, and M. Zarroug, "Hybrid frictional contact particles-in elements," Eur. J. Comput. Mech., vol. 11, no. 2-4, pp. 417-430, 2002. Volume Issue January
[12] M. Torkhani, $\mathrm{PhD}$ Thesis Contribution au développement numérique d'éléments de contact et modélisation de l'usure de structures minces École centrale de Lyon, 2008.

[13] N. Abbasi and K.-J. Bathe, "Stability and patch test performance of contact discretizations and a new solution algorithm," Comp. Struct., vol. 79, no. 16, pp. 1473-1486, 2001.

[14] F. Brezzi and M. Fortin, Mixed and Hybrid Finite Elements Methods, Berlin, Heidelberg: SpringerVerlag, 1991.

[15] P. Alart, F. Lebon, F. Quittau, and K. Rey, "Frictional contact problem in elastostatics: revisiting the uniqueness condition," in Proceedings of 2 nd Contact Mechanics International Symposium, and edited by M. Raous, J. Jean, and J. Moreau, New York and London: Plenum press, p. 63-70 1995.

[16] P. Ballard, "A counter-example to uniqueness in quasi-static elastic contact problems with small friction," Int. J. Eng. Sc., vol. 37, no. 2, pp. 163-178, 1999. pages

[17] P. Hild, "Solution multiplicity and stick configurations in continuous and finite element friction problems," Comp. Methods Appl. Mech. Eng., vol. 196, no. $1-3$, pp. $57-65,2006$. pages

[18] P. Alart, "Critères d injectivité et de surjectivité pour certaines applications de $\mathrm{Rn}$ dans lui même; application à la mécanique de contact," Math. Model. Numer. Anal., vol. 27, no. 2, pp. 203-222, 1993. pages

[19] P. Alart, "Méthode de newton généralisé en mécanique du contact," J. Math. Pures Et Appl., vol. 76, no. 1, pp. 83-108, 1997. pages

[20] P. Bussetta, D. Marceau, and J.-P. Ponthot, "The adapted augmented lagrangian method: a new method for the resolution of the mechanical frictional contact problem," Comput. Mech. Comput. Mech., vol. 49, no. 2, pp. 259-275, 2012. pages

[21] A. D. Kudawoo, Problèmes industriels de grandes dimensions en mécanique numérique du contact: performance, fiabilité et robustesse. $\mathrm{PhD}$ thesis, Université Aix Marseille, 2012.

[22] P. Pietrzak, and A. Curnier, "Large deformation frictional contact mechanics: continuum formulation and augmented lagrangian treatment," Comp Methods Appl. Mech. Eng., vol. 177, no. 3-4, pp. 351-381, 1999. pages

[23] P. Wriggers, Computational Contact Mechanics, 2nd ed., Berlin Heidelberg: Springer, 2006.

[24] M. Tur, F. Fuenmayor, and P. Wriggers, "A MortarBased Frictional Contact Formulation for Large Deformations Using Lagrange Multipliers, and Computer," Methods Appl. Mech. Eng., vol. 198, no. 37-40, pp. 2860-2873, 2009.

[25] J. C. Simo, "A framework for finite strain elastoplasticity based on maximum plastic dissipation and the multiplicative decomposition. Part I: Continuum formulation," Comput. Methods Appl. Mech. Eng, vol. 68, no. 1, p. 1, 1988.

[26] V. Yastrebov, Numerical Methods in Contact Mechanics, Hoboken, NJ: Wiley-ISTE,, 2013. 